\title{
Universal infrared conductivity of graphite
}

\author{
L.A. Falkovsky ${ }^{1,2}$ \\ ${ }^{1}$ L.D. Landau Institute for Theoretical Physics, Moscow 117334, Russia \\ ${ }^{2}$ Institute of the High Pressure Physics, Troitsk 142190, Russia
}

(Dated: November 7, 2018)

\begin{abstract}
The conductivity of graphite is analytically evaluated in the range of $0.1-1.5 \mathrm{eV}$, where the electron relaxation processes can be neglected, and the low energy excitations at the "Dirac" points are most essential. The value of conductivity calculated per one graphite layer is close to the universal conductivity of graphene. The features of the conductivity are explained in terms of singularities of the electron dispersion in graphite.

PACS numbers: 78.67.-n, 81.05.Bx, 81.05.Uw
\end{abstract}

Since the pioneering experimental investigations of a single atomic layer of graphite (graphene) $)^{1.2}$, its properties attract much attention. Among them, the optical response is of particular interest. Recently the transmittance of light throw the graphene monolayer has been measured ${ }^{3} \underline{\underline{-5}}$. The transmittance

$$
T=1-\pi \alpha
$$

was found to be frequency independent in a broad range of photon energy. The result of the experiments is remarkable because it involves the fine structure constant $\alpha$. It was discovered that the real part of the optical conductance of graphene takes the universal value

$$
G=\frac{e^{2}}{4 \hbar}
$$

which does not depend on any parameters of graphene. This value agrees perfectly with the calculations ${ }^{6.7}$ ignoring the Coulomb interactions between electrons. The agreement shows that the poorly screened Coulomb interaction does not play any role in graphene for infrared photon frequencies $\frac{8.9}{9}$.

The intermediate place between $2 \mathrm{~d}$ graphene and $3 \mathrm{~d}$ semiconductors belongs to multilayer graphenes 10 and graphite, which have a layered structure with the interlayer distance $c_{0}=3.35 \AA$ much larger than the nearestneighbor distance $a_{0}=1.42 \AA$ in the layer. In the study of graphite $\frac{11}{1}$, it was found that its optical conductivity per one layer is very closed to the universal conductivity of graphene and has evident peculiarities. The analytic calculation of the in-plane optical response of graphite done previously 12 has ignored coupling between layers and no peculiarities have appeared for the infrared region.

In the present paper, we evaluate analyticaly the conductance of graphite in the infrared region of the photon frequencies. It is known that the low energy electron excitations in graphene can be described very well with the Slonczewski-Weiss-McClure theory ${ }^{13}$. The largest parameter of the theory, $\gamma_{0}=3.1 \mathrm{eV}^{14}$, describes the electron dispersion for in-layer directions $\mathbf{k}$. If the photon energy is less than $\gamma_{0}$, we can use the linear expansion of the in-layer hopping term in the Hamiltonian and introduce the constant velocity parameter $v=10^{8} \mathrm{~cm} / \mathrm{s}$. The second parameter in the rang is the interlayer hopping $\gamma_{1}$ of the order of $0.4 \mathrm{eV}$ which is known from experiments on bilayer graphene $\frac{15,16}{}$. The parameters $\gamma_{3}$ and $\gamma_{4}$ give the corrections of the order of $10 \%$ to the in-layer velocity $v$. The electron-hole overlap of the order of 0.02 $\mathrm{eV}$ is determined by parameters $\gamma_{2}$ and $\gamma_{5}$ (see Fig. 2). Therefore, for the photon frequencies larger than $0.1 \mathrm{eV}$, we can neglect the terms with $\gamma_{2}$ and $\gamma_{5}$. Calculating such the integral property as conductivity in the region of the infrared frequencies between $0.1 \mathrm{eV}$ and $1.5 \mathrm{eV}$, we can, first, neglect the small parameters of the theory and, second, use the $k$-expansion of the in-layer hopping term. Our results have the evident analytic form.

In this approximation, the effective Hamiltonian writes near the K-G-H lines of the Brillouin zone in the simple form

$$
H(\mathbf{k})=\left(\begin{array}{cccc}
0 & k_{+} & \gamma(z) & 0 \\
k_{-} & 0 & 0 & 0 \\
\gamma(z) & 0 & 0 & k_{-} \\
0 & 0 & k_{+} & 0
\end{array}\right)
$$

determined only by two constants. One is $v=10^{8} \mathrm{~cm} / \mathrm{s}$ included in the definition of the in-plane momentum components, $k_{ \pm}=v\left(\mp i k_{x}-k_{y}\right)$, and another is the inter-layer interaction $\gamma_{1}$ involved in the function $\gamma(z)=2 \gamma_{1} \cos z$. The momentum component $z=k_{z} c_{0}$ is limited by the Brillouin half-zone, $0<z<\pi / 2$ in relative units.

The corresponding eigenenergies are

$$
\begin{gathered}
\varepsilon_{1,2}=\frac{\gamma(z)}{2} \pm \sqrt{\frac{1}{4} \gamma^{2}(z)+k^{2}}, \\
\varepsilon_{3,4}=-\frac{\gamma(z)}{2} \pm \sqrt{\frac{1}{4} \gamma^{2}(z)+k^{2}} .
\end{gathered}
$$

On the $\mathrm{K}-\mathrm{G}-\mathrm{H}$ lines, $k=0$, these equations determine two bands $\varepsilon_{1,4}= \pm \gamma(z)$ and two degenerate (electron and hole) bands with the energy $\varepsilon_{2,3}=0$. We have to emphasize that this degeneracy results from $C_{3 v}$ symmetry on the K-G-H line.

In order to calculate the conductivity, we use the general expression

$$
\sigma^{i j}(\omega)=\frac{2 i e^{2}}{(2 \pi)^{3}} \int d^{3} k \sum_{k, n \geq m}\left\{-\frac{d f}{d \varepsilon_{n}} \frac{v_{n}^{i} v_{n}^{j}}{\omega+i \nu}\right.
$$




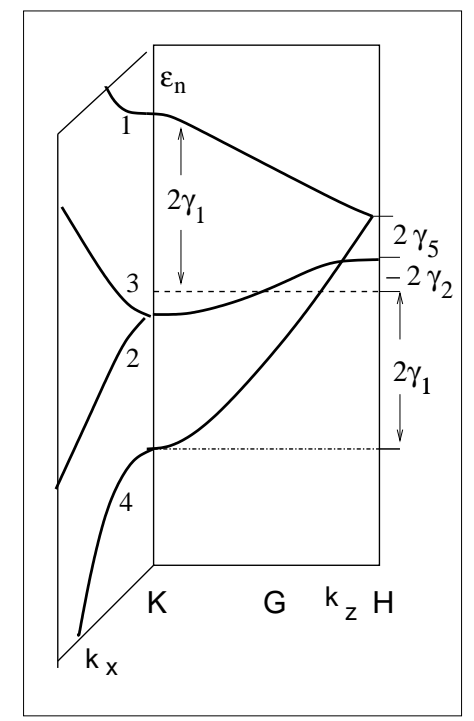

FIG. 1: The dispersion of the low energy electron bands in graphite.

$$
\left.+2 \omega \frac{v_{n m}^{i} v_{m n}^{j}\left\{f\left[\varepsilon_{n}(\mathbf{k})\right]-f\left[\varepsilon_{m}(\mathbf{k})\right]\right\}}{\left[\varepsilon_{m}(\mathbf{k})-\varepsilon_{n}(\mathbf{k})\right]\left\{(\omega+i \nu)^{2}-\left[\varepsilon_{n}(\mathbf{k})-\varepsilon_{m}(\mathbf{k})\right]^{2}\right\}}\right\}
$$

valid in the collisionless limit $\omega \gg \nu$, where $\nu$ is the collision rate of the carriers, $f(\varepsilon)=\left[\exp \left(\frac{\varepsilon-\mu}{T}\right)-1\right]^{-1}$ is the Fermi-Dirac distribution function, and the integral is over the Brillouin zone.

Here, the first term is the Drude-Boltzmann conductivity negligible for frequencies larger than the electron-hole overlap. The second term represents the optical interband transitions of electrons from the valence 2,4 to conductive 1,3 bands. The real part of the interband contributions into conductivity arises from the bypass around the pole at $\varepsilon_{n}(\mathbf{k})-\varepsilon_{m}(\mathbf{k})= \pm \omega$. The imaginary part is given by the principal value of the integral.

The velocity operator

$$
\mathbf{v}=\frac{\partial H(\mathbf{k})}{\partial \mathbf{k}}
$$

near the K-G-H lines is determined by the Hamiltonian (11). The corresponding matrix elements should be calculated in the representation, where the Hamiltonian has a diagonal form. The operator transforming the Hamiltonian to this form can be written as follows

$$
U=\left(\begin{array}{cccc}
\varepsilon_{1} / N_{1} & \varepsilon_{2} / N_{2} & -\varepsilon_{3} / N_{3} & -\varepsilon_{4} / N_{4} \\
k_{-} / N_{1} & k_{-} / N_{2} & -k_{-} / N_{3} & -k_{-} / N_{4} \\
\varepsilon_{1} / N_{1} & \varepsilon_{2} / N_{1} & \varepsilon_{3} / N_{3} & \varepsilon_{4} / N_{4} \\
k_{+} / N_{1} & k_{+} / N_{2} & k_{+} / N_{3} & k_{+} / N_{4}
\end{array}\right)
$$

where $N_{n}^{2}=2\left(\varepsilon_{n}^{2}+k^{2}\right)$. In this representation, the velocity operator

$$
U^{-1} \mathbf{v} U
$$

has the matrix elements

$$
\begin{gathered}
\mathbf{v}_{n n}=\partial \varepsilon_{n} / \partial \mathbf{k}, \\
\left.\mathbf{v}_{23}=2 i\left(\varepsilon_{3}-\varepsilon_{2}\right)\left(-k_{x} \mathbf{e}_{y}+k_{y} \mathbf{e}_{x}\right)\right] / N_{2} N_{3}, \\
\left.\mathbf{v}_{12}=2\left(\varepsilon_{1}+\varepsilon_{2}\right)\left(k_{x} \mathbf{e}_{x}+k_{y} \mathbf{e}_{y}\right)\right] / N_{1} N_{2}, \\
\left.\mathbf{v}_{14}=2 i\left(\varepsilon_{4}-\varepsilon_{1}\right)\left(-k_{x} \mathbf{e}_{y}+k_{y} \mathbf{e}_{x}\right)\right] / N_{1} N_{4},
\end{gathered}
$$

where $\mathbf{e}_{i}$ are the unit vectors directed along the coordinate axes. For the real part of conductivity, the integration in Eq. (2) is easily taken at zero temperatures $T=0$ in cylindrical coordinates $\left(k_{z}, k, \phi\right)$ over the angle $\phi$ and over $k$ with the help of the $\delta$-function, $(\omega-x+i \nu)^{-1} \rightarrow-i \pi \delta(\omega-x)$. One obtains for contributions of the transitions between the corresponding valence and conduction bands into the diagonal components of conductivity (off-diagonal ones equal zero) the following integrals over $z=k_{z} / c_{0}$ :

$$
\begin{gathered}
\operatorname{Re} \sigma_{23}=\frac{e^{2}}{4 \pi \hbar c_{0}} \int_{0}^{\pi / 2} d z \frac{2 \gamma(z)+\omega}{\gamma(z)+\omega}, \\
\operatorname{Re} \sigma_{21}=\frac{e^{2}}{4 \pi \hbar c_{0}} \int_{0}^{\pi / 2} d z \frac{\gamma^{2}(z)}{\omega^{2}} \theta[\omega-\gamma(z)], \\
\operatorname{Re} \sigma_{41}=\frac{e^{2}}{4 \pi \hbar c_{0}} \int_{0}^{\pi / 2} d z \frac{2 \gamma(z)-\omega}{\gamma(z)-\omega} \theta[\omega-2 \gamma(z)], \\
\sigma_{43}=\sigma_{21},
\end{gathered}
$$

where $\gamma(z)=2 \gamma_{1} \cos z$ and $\theta(x)$ is the step function.

It is evident from Eqs. (3) (see also Fig. 2) that the conductivity $\sigma_{23}$ tends to $e^{2} / 4 \hbar c_{0}$ at the low frequencies $\omega \ll 2 \gamma_{1}$, whereas other contributions go to zero in the limit of low frequencies. At larger frequencies $\omega \gg 2 \gamma_{1}$, the total conductivity (the sum of $\sigma_{23}$ and $\sigma_{41}$ ) tends again to $e^{2} / 4 \hbar c_{0}$. Therefore, $\sigma_{0}=e^{2} / 4 \hbar c_{0}$ can be considered as the universal conductivity of graphite, where $e^{2} / 4 \hbar$ is the conductivity of monolayer graphene and the factor $1 / c_{0}$ is the number of the layers per the length unit in the z-direction of graphite. Integrating in Eqs. (3), we get finally

$$
\operatorname{Re} \frac{\sigma_{23}}{\sigma_{0}}=1-\frac{2 t}{\pi \sqrt{t^{2}-1}} \arctan \sqrt{\frac{t-1}{t+1}}, t>1
$$

$$
\begin{gathered}
\operatorname{Re} \frac{\sigma_{23}}{\sigma_{0}}=1-\frac{t}{\pi \sqrt{1-t^{2}}} \ln \frac{\sqrt{1+t}+\sqrt{1-t}}{\sqrt{1+t}-\sqrt{1-t}}, t<1, \\
\operatorname{Re} \frac{\sigma_{21}}{\sigma_{0}}=\frac{1}{4 t^{2}} \begin{cases}1, & t>1, \\
1-\frac{2}{\pi}\left(\arccos t+t \sqrt{1-t^{2}}\right), & t<1 .\end{cases}
\end{gathered}
$$

$$
\operatorname{Re} \frac{\sigma_{41}}{\sigma_{0}}=1-\frac{2 t}{\pi \sqrt{t^{2}-1}} \arctan \sqrt{\frac{t+1}{t-1}}, t>2
$$




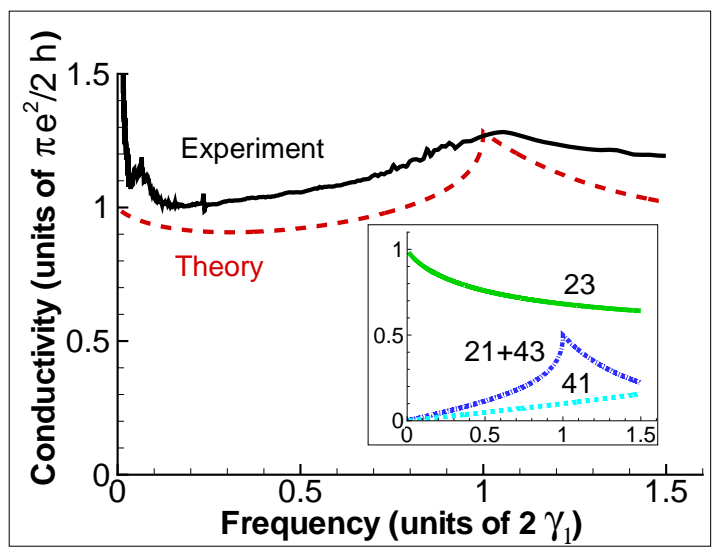

FIG. 2: The real part of the graphite conductivity per layer (in units of $e^{2} / 4 \hbar$ ) versus the frequency (in units of $2 \gamma_{1}=$ $0.84 \mathrm{eV}$ ); the experimental data ${ }^{11}$ are shown in the solid line, results of the present theory in the dashed line. The insert shows the contributions of various electron transitions.

$$
\begin{array}{r}
\operatorname{Re} \frac{\sigma_{41}}{\sigma_{0}}=1-\frac{2 z_{1}}{\pi}-\frac{2 t}{\pi \sqrt{t^{2}-1}}\left[\arctan \sqrt{\frac{t+1}{t-1}}\right. \\
\left.-\arctan \left(\sqrt{\frac{t+1}{t-1}} \tan \frac{z_{1}}{2}\right)\right], 1<t<2, \\
\operatorname{Re} \frac{\sigma_{41}}{\sigma_{0}}=1-\frac{2 z_{1}}{\pi}+\frac{t}{\pi \sqrt{1-t^{2}}}\left[\ln \frac{\sqrt{1+t}+\sqrt{1-t}}{\sqrt{1+t}-\sqrt{1-t}}\right. \\
\left.+\ln \frac{\sqrt{1+t} \tan \frac{z_{1}}{2}-\sqrt{1-t}}{\sqrt{1+t} \tan \frac{z_{1}}{2}+\sqrt{1-t}}\right], t<1,
\end{array}
$$

where $t=\omega / 2 \gamma_{1}$ and $z_{1}=\arccos (t / 2)$.

The peculiarity as a kink can be seen in Fig. 2, The expression (5) shows that this kink is located at $\omega=$ $2 \gamma_{1}$. Taking into account the kink position $\omega=0.84 \mathrm{eV}$ determined experimentally, the value of $\gamma_{1}=0.42 \mathrm{eV}$ is found in excellent agreement with experiments on bilayer graphene.

The contributions of the electron interband transitions into the imaginary part of conductivity can be integrated over $k$ at the zero temperature. The results are obtained in the form of integrals over $k_{z}$

$$
\operatorname{Im} \frac{\sigma_{23}}{\sigma_{0}}=\frac{2}{\pi^{2}} \int_{0}^{\pi / 2} d z \frac{\omega \gamma(z)}{\gamma^{2}(z)-\omega^{2}} \ln [\gamma(z) / \omega]
$$

$\operatorname{Im} \frac{\sigma_{21}}{\sigma_{0}}=\frac{1}{\pi^{2}} \int_{0}^{\pi / 2} d z \frac{\gamma(z)}{\omega}\left(2+\frac{\gamma(z)}{\omega} \ln \frac{|\gamma(z)-\omega|}{\gamma(z)+\omega}\right)$

$$
\begin{array}{r}
\operatorname{Im} \frac{\sigma_{41}}{\sigma_{0}}=\frac{1}{\pi^{2}} \int_{0}^{\pi / 2} \\
d z\left(\frac{2 \gamma(z)-\omega}{\gamma(z)-\omega} \ln |2-\omega / \gamma(z)|\right. \\
\left.-\frac{2 \gamma(z)+\omega}{\gamma(z)+\omega} \ln (2+\omega / \gamma(z))\right)
\end{array}
$$

and shown in Fig. 3. Here, the peculiarity looks like a threshold at $\omega=2 \gamma_{1}$ and it is more clearly marked

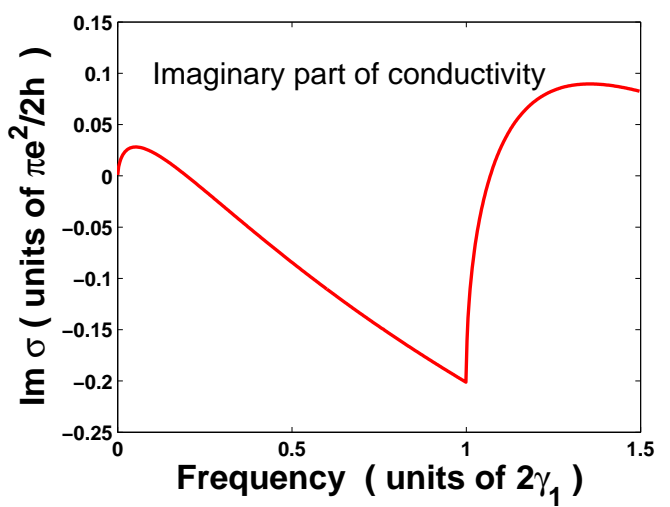

FIG. 3: The imaginary part of the graphite conductivity per layer (in units of $e^{2} / 4 \hbar$ ) versus the frequency (in units of $\left.2 \gamma_{1}=0.84 \mathrm{eV}\right)$.

in comparison with the kink in the real conductivity. Both peculiarities result due to the electron transitions between the bands $2 \rightarrow 1$ and $4 \rightarrow 3$. We should emphasize that the peculiarities become broader with the temperatures and the collision processes included.

So far the in-layer conductivity was considered. The estimate of the inter-layer conductivity can be also done. Since the conductivity is determined by the ratio of the corresponding velocities squared, we have to write

$$
v_{z}=\frac{\partial \varepsilon_{3}}{\partial k_{z}} \sim \gamma_{1} c_{0} \sin \left(k_{z} c_{0}\right) .
$$

Then, integrating over $k_{z}$, we get

$$
\sigma_{z} / \sigma_{0} \sim\left(\gamma_{1} c_{0} / \hbar v\right)^{2} / 2 \sim 0.05 .
$$

In conclusion, our calculations reveals that the optical conductance of graphite can be estimated for frequencies between 0.1 and $1.5 \mathrm{eV}$ multiplying the graphene conductivity $e^{2} / 4 \hbar$ by the number of the layers $1 / c_{0}$ per the length unit. The Drude-Boltzmann contribution is essential at lower frequencies, whereas others interband transitions, e.g. at the M point of the Brillouin zone contribute into the conductivity at higher frequencies. The similar estimate are applicable for other graphite materials such as nanoribbons. The kink in the real part of conductivity and the threshold in the imaginary part appear at the frequency $\omega=2 \gamma_{1}$ determined by the interlayer coupling. The sharpness of the features are smeared with the relaxation processes and temperatures included.

This work was supported by the Russian Foundation for Basic Research (grant No. 10-02-00193-a) and by the SCOPES grant IZ73Z0_128026 of the Swiss NSF. The author is grateful to the Max Planck Institute for the Physics of Complex Systems for hospitality in Dresden. 
1 K.S. Novoselov et al., Science, 306, 666 (2004), K.S. Novoselov et al., Nature, 438, 197 (2005).

${ }^{2}$ Y. Zhang, J.P. Small, M.E.S. Amory, P.Kim, Phys. Rev. Lett. 94, 176803 (2005).

3 R.R. Nair, P. Blake, A.N. Grigorenko, K.S. Novoselov, T.J. Booth, T. Stauber, N.M.R. Peres, A.K. Geim, Science 320, 5881 (2008).

4 Z.Q. Li, E.A. Henriksen, Z. Jiang, Z. Hao, M.C. Martin, P. Kim, H.L. Stormer, D.N. Basov, Nature Physics 4, 532 (2008).

${ }^{5}$ K.F. Mak, M.Y. Sfeir, Y. Wu, C.H. Lui, J.A. Misewich, and Tony F. Heinz, Phys. Rev. Lett. 101, 196405 (2008).

6 V.P. Gusynin, S.G. Sharapov, and J.P. Carbotte, Phys. Rev. Lett. 96, 256802 (2006).

7 L.A. Falkovsky and A.A Varlamov, Eur. Phys. J. B 56, 281 (2007).
8 E.G. Mishchenko, Europhys. Lett. 83, 17005 (2008).

9 D.E. Sheehy and J. Scmalian, arXiv:0906.5164vl

10 M. Koshino, T. Ando, Sol. St. Comm. 149, 1123 (2009).

11 A.B. Kuzmenko, E. van Heumen, F. Carbone, and D. van der Marel, Phys. Rev. Lett. 100, 117401 (2008).

12 T.G. Pedersen, Phys. Rev. B 67, 113106 (2003).

13 J.W. McClure, Phys. Rev. 108, 612 (1957); J.C. Slonczewski and P.R. Weiss, Phys. Rev. 109, 272 (1958);

14 B. Partoens and F.M. Peeters, Phys. Rev. B 74, 075404 (2006).

15 A.B. Kuzmenko, I. Crassee,, D. van der Marel, P. Blake, and K.S. Novoselov, Phys. Rev. 80, 165406 (2009).

16 Z.Q. Li, E.A. Henriksen, Z. Jiang, Z. Hao, M.C. Martin, P. Kim, H.L. Stormer, and D.N. Basov, Phys. Rev. Lett. 102, 037403 (2009). 